\title{
Optical Properties of Gallium-Doped Zinc Oxide-A Low-Loss Plasmonic Material: First-Principles Theory and Experiment
}

\author{
Jongbum Kim, ${ }^{1}$ Gururaj V. Naik, ${ }^{1}$ Alexander V. Gavrilenko, ${ }^{2}$ Krishnaveni Dondapati, ${ }^{2}$ Vladimir I. Gavrilenko, ${ }^{2}$ \\ S. M. Prokes, ${ }^{3}$ Orest J. Glembocki, ${ }^{3}$ Vladimir M. Shalaev, ${ }^{1}$ and Alexandra Boltasseva ${ }^{1}$ \\ ${ }^{1}$ Birck Nanotechnology Center and School of Electrical \& Computer Engineering, Purdue University, \\ West Lafayette, Indiana 47907, USA \\ ${ }^{2}$ Center for Materials Research, Norfolk State University, 700 Park Avenue, Norfolk, Virginia 23504, USA \\ ${ }^{3}$ Electronic Science Division, Naval Research Laboratory, Washington, D.C. 20375, USA
}

(Received 26 July 2013; published 31 December 2013)

\begin{abstract}
Searching for better materials for plasmonic and metamaterial applications is an inverse design problem where theoretical studies are necessary. Using basic models of impurity doping in semiconductors, transparent conducting oxides (TCOs) are identified as low-loss plasmonic materials in the near-infrared wavelength range. A more sophisticated theoretical study would help not only to improve the properties of TCOs but also to design further lower-loss materials. In this study, optical functions of one such TCO, gallium-doped zinc oxide (GZO), are studied both experimentally and by first-principles density-functional calculations. Pulsed-laser-deposited GZO films are studied by the x-ray diffraction and generalized spectroscopic ellipsometry. Theoretical studies are performed by the total-energy-minimization method for the equilibrium atomic structure of GZO and random phase approximation with the quasiparticle gap correction. Plasma excitation effects are also included for optical functions. This study identifies mechanisms other than doping, such as alloying effects, that significantly influence the optical properties of GZO films. It also indicates that ultraheavy $\mathrm{Ga}$ doping of $\mathrm{ZnO}$ results in a new alloy material, rather than just degenerately doped $\mathrm{ZnO}$. This work is the first step to achieve a fundamental understanding of the connection between material, structural, and optical properties of highly doped TCOs to tailor those materials for various plasmonic applications.
\end{abstract}

DOI: 10.1103/PhysRevX.3.041037

Subject Areas: Metamaterials, Plasmonics

\section{INTRODUCTION}

In the past decade, there have been many breakthroughs in the field of plasmonics and metamaterials that have enabled optical devices with unprecedented functionalities [1-4]. Many of these devices have remained in research laboratories without being realized in commercial production lines because of many technological hurdles. One such major problem haunting plasmonic and metamaterial devices is the high optical losses in plasmonic building blocks [5]. Typical plasmonic components such as gold or silver nanostructures exhibit large optical loss in the visible and near-infrared regions. Additionally, gold and silver have other drawbacks, such as difficulty in fabricating nanostructures, incompatibility with well-established processes like silicon complementary metal-oxide-semiconductor, and relatively low thermal and/or chemical stabilities. Hence, there is a need for finding better alternatives to gold and silver [5].

Finding a low-loss metallic component for a given plasmonic or metamaterial application is a complex

Published by the American Physical Society under the terms of the Creative Commons Attribution 3.0 License. Further distribution of this work must maintain attribution to the author $(s)$ and the published articles title, journal citation, and DOI. inverse-design problem. Hence, various simplified approaches have been attempted to produce low-loss metallic components. Given the fact that so far it has not been possible to realize a bulk zero-loss metal [6], reducing losses in plasmonic materials to a tolerable extent is a practical solution that is being pursued by many research groups (for a recent review, see Ref. [7] and references therein). One of the approaches is to tailor the carrier concentration of the plasmonic material to a moderate value [8]. Optical loss characterized by the imaginary part of permittivity is proportional to the carrier concentration of a plasmonic material. Hence, reducing carrier concentration of metals to a moderate value is one of the approaches in achieving low-loss plasmonic material. Using this approach, heavily doped semiconductors such as indium tin oxide (ITO), aluminum-doped zinc oxide (AZO), and gallium-doped zinc oxide (GZO) are shown to be low-loss plasmonic materials in the near infrared [9-11]. Recently, indium-doped cadmium oxide (ICO), another type of TCO, was also demonstrated as a low-loss plasmonic material in the near infrared [12]. TCOs exhibit losses nearly 5 times smaller than that of the best metal (Ag) in the near-infrared range, where they turn out to be plasmonic. Figure 1 plots the optical loss or imaginary permittivity $\left(\varepsilon^{\prime \prime}\right)$ of AZO, GZO, and ITO, along with that of noble metals, gold, and 


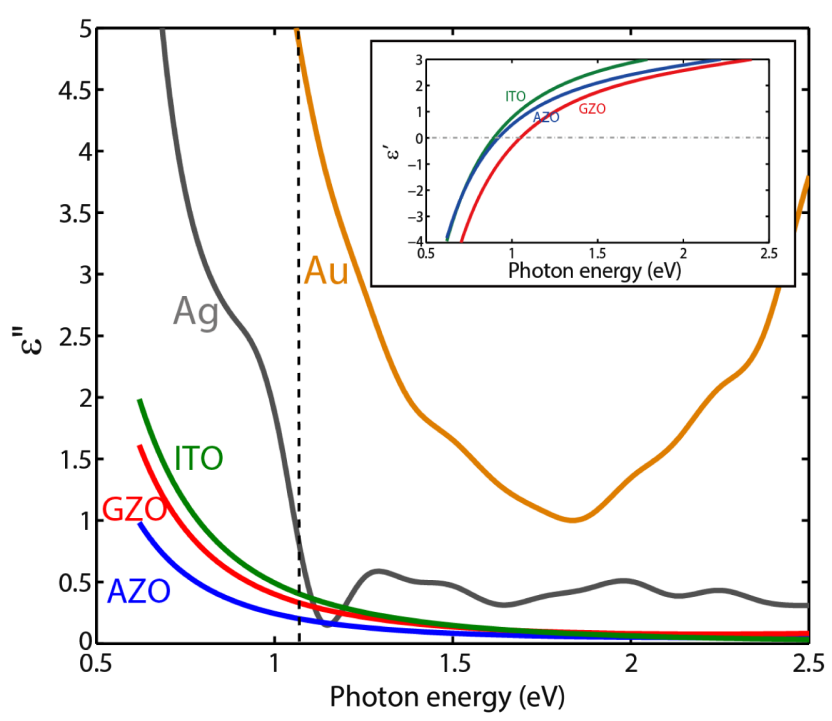

FIG. 1 (color online). Optical loss or imaginary part of permittivity of three transparent conducting oxides: ITO, AZO, and GZO, compared with that of gold and silver. Optical constants of thin films of AZO, GZO, and ITO are from Ref. [13], and those of gold and silver are from Ref. [14]. The inset shows the real part of permittivity of AZO, GZO, and ITO. The vertical dashed line corresponds to the photon energy where real permittivity of GZO crosses zero.

silver. It may be noted that smaller imaginary permittivity or optical loss does not necessarily mean better performance of a plasmonic or metamaterial device.

In general, the device performance depends on both the real and imaginary parts of permittivity. The performance of plasmonic devices can be quantified based on the figure of merit (FoM), which forms a common platform to evaluate the performance of various materials used in different applications over a wide frequency band, as suggested in Ref. [15]. Adopting the general FoM definition for localized surface-plasmon resonance (LSPR) applications as $|\operatorname{Re}(\varepsilon)| / \sqrt{\operatorname{Im}(\varepsilon)}$, the efficiency of various plasmonic materials can be evaluated [15]. The same FoM holds for any of the surface plasmon polariton waveguiding applications as well. Figure 2 plots this quantity for GZO, gold, and silver. Generally speaking, gold and silver outperform TCOs for these applications. Nevertheless, TCOs are good alternatives in the near infrared for simple device geometries [7]. Figure 2 also plots the FoM for transformation optics (TO) devices, which can be defined as the ratio of the real part of the refractive index $\left(n^{\prime}\right)$ to the imaginary part $\left(n^{\prime \prime}\right)$. From this plot, we can clearly see that TCOs are promising candidates as plasmonic materials in the near infrared for nonresonant applications such as TO devices. Devices such as epsilon-near-zero and hyperbolic metamaterials do benefit significantly by using TCOs as their plasmonic components.

The optical properties of TCOs may be further improved if their electronic band structure is engineered to achieve

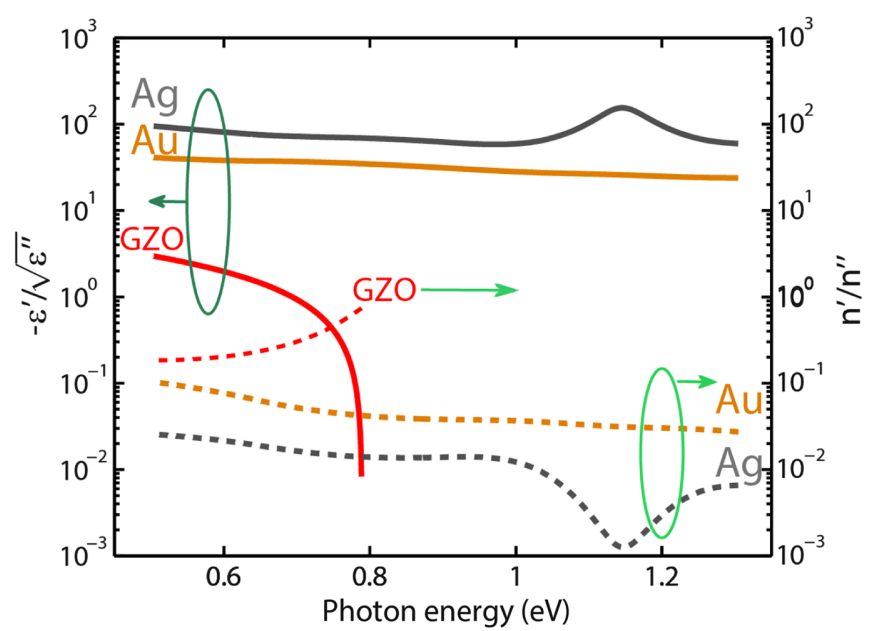

FIG. 2 (color online). FoM of LSPR and surface plasmon polariton waveguiding devices (solid lines) and TO devices (dashed lines) calculated for GZO, gold, and silver as plasmonic materials.

lower optical loss. Engineering the electronic band structure of materials to design plasmonic materials was demonstrated earlier with alkali-noble intermetallics and gold-cadmium alloys [16,17]. This approach requires theoretical studies using techniques such as density functional theory (DFT) in order to predict the optical properties of the "designer" plasmonic material. This approach may be applied to TCOs, and many other good plasmonic materials as well, to improve their optical properties and also gain better insight into solving the complex inverse design problem of finding low-loss plasmonic materials.

More detailed analyses on a comparison study of alternative materials can be found in Sec. 8 of Ref. [7]. Analyses have shown that TCOs are promising candidates as plasmonic materials in the near-infrared and longer wavelengths for devices based on epsilon-near-zero (ENZ), hyperbolic metamaterials, localized surface-plasmon resonance, and tunable devices.

In this work, we apply electronic band-structure calculations to study the optical properties of GZO. Among the three TCOs considered, GZO can be doped to achieve the highest carrier concentration in spite of the smaller solidsolubility limit of $\mathrm{Ga}$ in $\mathrm{ZnO}$ [18]. The optical properties of heavily doped GZO possess nontrivial dependence on the doping concentration, especially when doped as close to the solid-solubility limit as possible. Though there are many reports that study the electronic or optical properties of heavily doped GZO films [19-22], it is not well understood how the carrier concentration, crystallinity of films, and alloying effects influence the optical properties. Here, we use first-principles DFT to study the influence of each of these factors on the optical properties of plasmonic GZO films. Theoretical studies are supported with experimental studies on high-quality $n$-type wurtzite $\mathrm{ZnO}(w-\mathrm{ZnO})$ polycrystalline films doped with gallium. 
TABLE I. Typical deposition parameters for $3 \mathrm{wt} \%$ and $6 \mathrm{wt} \%$ GZO.

\begin{tabular}{lcc}
\hline \hline Doping density & $3 \mathrm{wt} \%$ & $6 \mathrm{wt} \%$ \\
\hline Ratio of pulses $\left(\mathrm{ZnO}: \mathrm{Ga}_{2} \mathrm{O}_{3}\right)$ & $60: 1$ & $30: 1$ \\
Ablation rate $(\mathrm{Hz})$ & 1 & 1 \\
Oxygen partial pressure (Torr) & $10^{-3}$ & $10^{-3}$ \\
\hline \hline
\end{tabular}

First-principles DFT calculations are incorporated to study the electron energy structure and optical functions of heavy Ga-doped wurtzite $\mathrm{ZnO}$ crystals. The ground states of the systems are obtained by the total energy minimization method within the local density approximation (LDA). The effects of Ga doping on the electron energy structure and optical functions are presented.

\section{SAMPLE PREPARATION AND CHARACTERIZATION}

Pulsed laser deposition (PLD) is a growth technique for thin films by condensation of laser plasma ablated from multiple targets excited by the high-energy laser pulses. In our studies, we employed PLD (PVD Products, Inc.) using a $\mathrm{KrF}$ excimer laser (Lambda Physik $\mathrm{GmbH}$ ) operating at a wavelength of $248 \mathrm{~nm}$ for source material ablation. The $\mathrm{Ga}_{2} \mathrm{O}_{3}$ and $\mathrm{ZnO}$ targets were purchased from the Kurt J. Lesker Corp., with purities of $99.99 \%$ or higher. The films were deposited at $70^{\circ} \mathrm{C}$, and the energy density of the laser beam at the target surface was maintained at $1.5 \mathrm{~J} / \mathrm{cm}^{2}$. $\mathrm{ZnO}$ and $\mathrm{Ga}_{2} \mathrm{O}_{3}$ targets were repeatedly ablated in a cycle consisting of a predetermined number of pulses on each of the two targets. The $\mathrm{Ga}_{2} \mathrm{O}_{3}$ doping concentration was varied in the range from 0 to $6 \mathrm{wt} \%$ by varying the number of ablation pulses on the $\mathrm{Ga}_{2} \mathrm{O}_{3}$ target within each cycle. In order to ensure homogeneous mixing of $\mathrm{Ga}_{2} \mathrm{O}_{3}$ in $\mathrm{ZnO}$, the number of ablation pulses on each target during each cycle was kept small. Within each ablation cycle, only one pulse of laser were assigned to the $\mathrm{Ga}_{2} \mathrm{O}_{3}$ target, while a variable number of pulses (depending on the composition required) were assign to $\mathrm{ZnO}$ target. If an effective thickness of the resultant film deposited in each cycle was computed, the total thickness would be less than $2 \mathrm{~nm}$. This ensures that the films are homogeneous and uniformly doped. Undoped films and films with 3 and $6 \mathrm{wt} \% \mathrm{Ga}$ doping were prepared. The typical deposition parameters for $3 \mathrm{wt} \%$ and $6 \mathrm{wt} \%$ gallium doping are listed in Table I.

The dependence of the optical properties of the GZO films on the doping concentration was studied using a spectroscopic ellipsometer (M-2000, J.A. Woollam Co.) in the spectral region from 0.73 to $6.52 \mathrm{eV}$. In order to retrieve numerical data from the measured optical functions, we used a semi-empirical model describing the contributions of both free and bound electrons $[5,8]$. The Drude oscillator model is used to describe contributions of the conduction electrons that behave as free electron gas. Within the perturbation theory, optical response from the bound electrons is well described by the oscillator model containing a set of Lorentz- or Gaussian-type single oscillators [23]. In this work, we use the Gaussian oscillator model to describe the contributions of the bound electrons. Because of the structural disorder in solid alloys, the optical functions contain a random component of optical response well described by a Gaussian distribution [24]. The following equation set presents the Drude-plus-Gaussian oscillator model:

$$
\begin{gathered}
\epsilon(\omega)=\epsilon_{\infty}-\epsilon_{\text {Drude }}+\sum_{m=1}^{n} \epsilon_{\text {Gaussian }}\left(\omega_{m}, \sigma_{m}\right) \quad(n=2), \\
\epsilon_{\text {Gaussian }}\left(\omega_{m}, \sigma_{m}\right)=\epsilon_{m 1}+i \epsilon_{m 2}, \\
\epsilon_{m 1}=\frac{2}{\pi} P \int_{0}^{\infty} \frac{\xi \epsilon_{m 2}(\xi)}{\xi^{2}-\omega^{2}} d \xi, \\
\epsilon_{m 2}=A_{m} e^{-\frac{\left(\omega-\omega_{m}\right)^{2}}{\sigma_{m}}}+A_{m} e^{-\frac{\left(\omega+\omega_{m}\right)^{2}}{\sigma_{m}}}\left(\sigma_{m}=\frac{\Gamma_{m}}{2 \sqrt{\ln (2)}}\right), \\
\epsilon_{\text {Drude }}=\frac{\omega_{p}^{2}}{\omega^{2}+i \Gamma_{p} \omega},
\end{gathered}
$$

where $\epsilon_{\infty}$ is the macroscopic dielectric permittivity, $\omega_{p}$ is the plasma frequency, $\Gamma_{p}$ is the carrier relaxation rate, $A_{m}$ is proportional to the oscillator strength, $\omega_{m}$ is the central frequency of interband electronic transitions represented by the Gaussian oscillator, and $\Gamma_{m}$ is the broadening of the Gaussian oscillator. The extracted parameters for GZO thin films with different concentrations of gallium (undoped, $3 \mathrm{wt} \%$ and $6 \mathrm{wt} \%$ ) are summarized in Table II, and Fig. 3 shows the dielectric function measured for GZO thin films. In our previous study [10], the $6 \mathrm{wt} \%$ was the optimum value of the doping rate to achieve the lowest drudedamping coefficient. By increasing the doping rate in the range below the optimum value, the crossover frequency becomes lower because of the increase of carrier concentration, and the optical loss becomes lower because of the improved crystallinity.

TABLE II. Extracted Drude + Gaussian oscillator parameter.

\begin{tabular}{lccc}
\hline \hline Doping rate & Undoped & $3 \mathrm{wt} \%$ & $6 \mathrm{wt} \%$ \\
\hline$\epsilon_{\infty}$ & 2.812 & 2.557 & 2.439 \\
$\omega_{p}$ & 0 & 1.508 & 1.901 \\
$\Gamma_{p}$ & - & 0.159 & 0.092 \\
$A_{1}$ & 1.742 & 1.630 & 1.427 \\
$A_{2}$ & 1.865 & 2.101 & 1.676 \\
$\Gamma_{1}$ & 0.596 & 1.036 & 0.764 \\
$\Gamma_{2}$ & 3.222 & 2.922 & 3.251 \\
$\omega_{1}$ & 3.611 & 4.335 & 4.371 \\
$\omega_{2}$ & 5.905 & 6.603 & 5.825 \\
\hline \hline
\end{tabular}




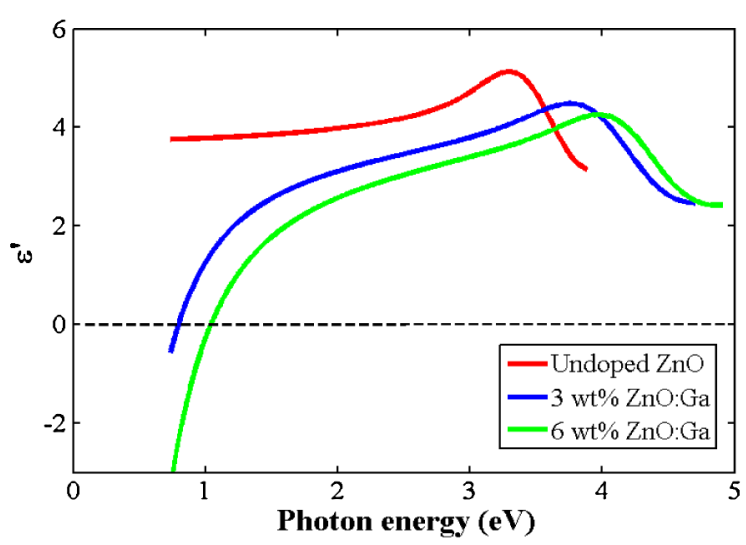

(a)

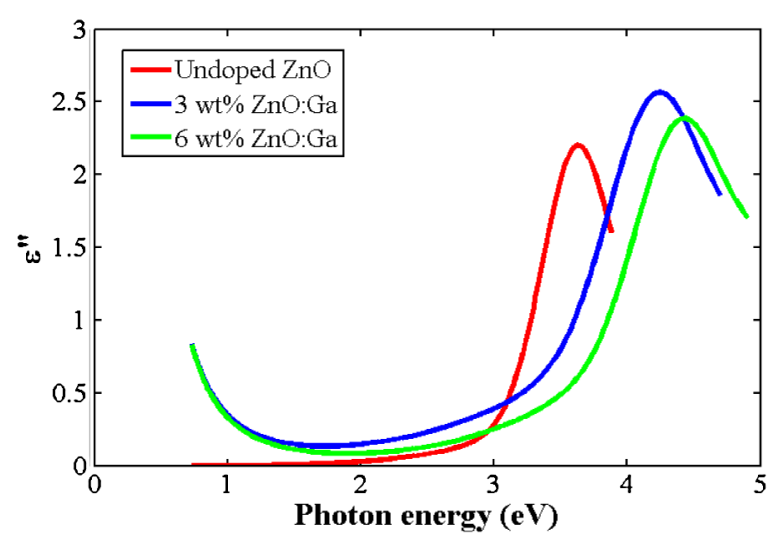

(b)

FIG. 3 (color online). Real and imaginary parts of the dielectric function spectra of $\mathrm{w}-\mathrm{ZnO}$ and GZO thin films.

The structural properties of $\mathrm{ZnO}$ and GZO thin films were examined using X-ray diffraction (Panalytical X'Pert Pro MRD) studies with a parallel beam configuration and $\mathrm{CuK} \alpha$ excitation. Figure 4 shows x-ray diffraction data $(\omega-2 \theta$ scans) of the films with different concentrations of

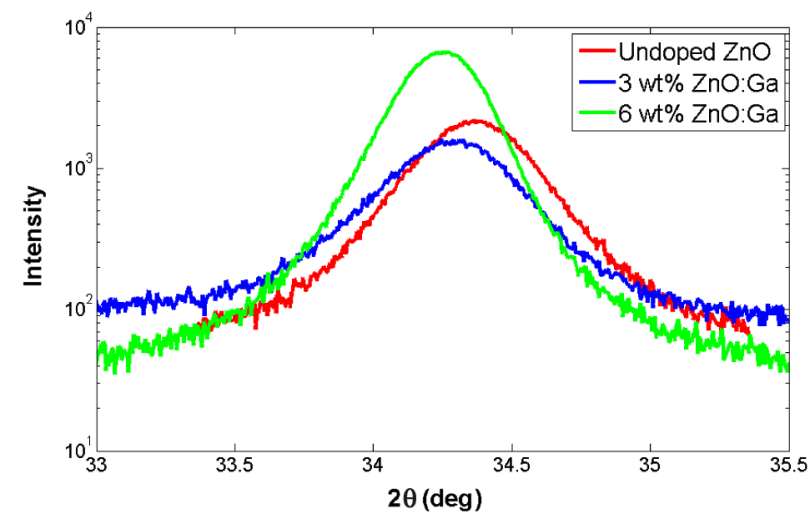

FIG. 4 (color online). X-ray diffraction $(\omega-2 \theta)$ patterns for $\mathrm{ZnO}, 3 \mathrm{wt} \% \mathrm{GZO}$, and $6 \mathrm{wt} \% \mathrm{GZO}$ thin films. The peak shown in the figure corresponds to 002 reflection.

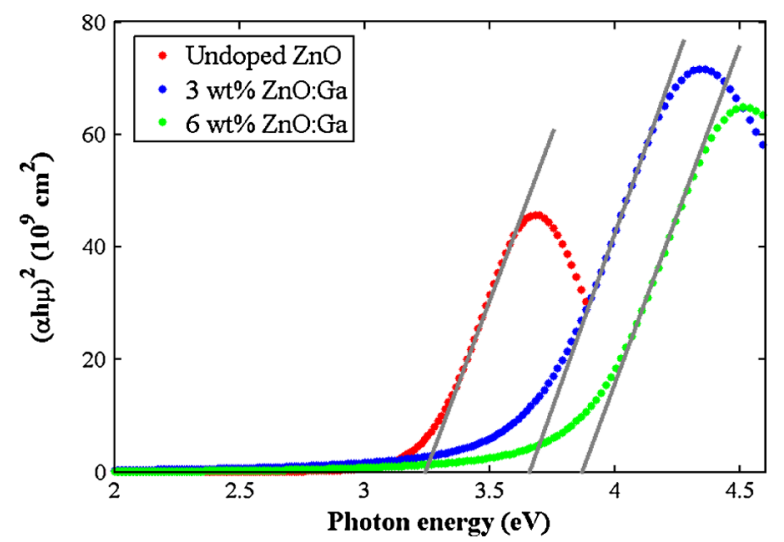

FIG. 5 (color online). Square of the absorption coefficient as a function of photon energy for $\mathrm{ZnO}, 3 \mathrm{wt} \% \mathrm{GZO}$ and $6 \mathrm{wt} \% \mathrm{GZO}$ thin films. $E_{g}$ proportional to $(\alpha \hbar \omega)^{2}$ is calculated by fitting linear line to spectra $E_{g}$ (Undoped $\mathrm{ZnO}$ ):3.25 eV, $E_{g}$ (3 wt\% GZO):3.66 eV and $E_{g}(6 \mathrm{wt} \% \mathrm{GZO}): 3.25 \mathrm{eV}$

gallium (undoped, $3 \mathrm{wt} \%$ and $6 \mathrm{wt} \%$ ). The GZO films deposited are polycrystalline and strongly oriented along the $c$ axis normal to the quartz substrate surface. Strong orientation of grains resulted in 002 reflection appearing around $34.5^{\circ}$ as the only dominant peak observed [25]. In this work, only a 002 reflection peak is considered for subsequent analyses (see Fig. 4).

The optical band gap in GZO films can be determined from the spectral dependence of the absorption coefficient $(\alpha)$. In the direct-gap semiconductors such as $\mathrm{ZnO}$, the absorption coefficient $\alpha$ and optical band gap $\left(E_{g}\right)$ are related by Eq. (6) [26]:

$$
\alpha \hbar \omega=A\left(\hbar \omega-E_{g}\right)^{\frac{1}{2}},
$$

where $\hbar \omega$ is the incident photon energy and $A$ is a proportionality constant. The values of $E_{g}$ are extracted from the plot of $\alpha^{2}$ vs $\hbar \omega$, as shown in Fig. 5. The $E_{g}$ values are obtained from the intercept of the $\alpha^{2}$ asymptote on the horizontal axis. This procedure results in values of $E_{g}$ of $3.25 \mathrm{eV}$ for undoped $\mathrm{ZnO}$, and $3.66 \mathrm{eV}$ and $3.89 \mathrm{eV}$ for $3 \mathrm{wt} \%$ and $6 \mathrm{wt} \%$ GZO films, respectively.

\section{THEORETICAL STUDIES}

Heavy Ga-doped wurtzite $\mathrm{ZnO}$ crystals are modeled by the first-principles pseudopotential theory using the supercell method. Equilibrium atomic structures of w-GZO are obtained from the total energy minimization method within DFT using ab initio pseudopotentials [27]. We employ the supercell approach to model the substitutional defects; our unit cell of GZO- $2 \times 2 \times 2$ contains 32 atoms that correspond to a new $\mathrm{Zn}_{1-x} \mathrm{Ga}_{x} \mathrm{O}$ compound with an atomic $\mathrm{Ga}$ concentration of about 6 at.\% $(x=0.067)$.

Ultrasoft pseudopotentials including $3 d$ transition metal states in the valence band are implemented with an energy 
cutoff of $420 \mathrm{eV}$. For reliable total energy convergence, $N_{\mathbf{k}}$ in the irreducible part of the brillouin zone (BZ) have been used up to $400 \mathbf{k}$ point. With the chosen $N_{\mathbf{k}}$ and energy cutoff value, the total energy converges within a few percent. However, the density of states (DOS) convergence tests indicate that an accuracy within 5\% (or better) has been achieved for DOS by using at least $1270 \mathbf{k}$ points, as in the present work for DOS and optics.

Optical functions of GZO are calculated within the random phase approximation, as described before $[23,28]$. Our calculated energy gap of $0.70 \mathrm{eV}$ in bulk $\mathrm{ZnO}$ is substantially lower than the experimental value of $3.25 \mathrm{eV}$ [29]. However, it agrees well with previously reported DFT-LDA and DFT-GGA gap values [30-32]. The widely used scissor-operator correction is implemented in this calculation in order to account for the energy-gap underestimate and enable better comparison with experimental data $[28,31,32]$. With this method, the calculated optical spectra shift to blue.

\section{RESULTS AND DISCUSSION}

Equilibrium LDA hexagonal lattice constants of bulk w-ZnO and doped GZO crystals are given in Table III.

Substitution of $\mathrm{Ga}$ on the $\mathrm{Zn}$ site $\left(\mathrm{Ga}_{\mathrm{Zn}}\right)$ creates a singly occupied singlet defect state in the conduction band and thus acts as a donor in $\mathrm{ZnO}$. The relaxed structure shows that in the $1^{+}$state, Ga-O distances are approximately $5 \%$ to $7 \%$ shorter than corresponding values in bulk $\mathrm{ZnO}$. These values agree with those reported before for the local contraction in GZO [32]. However, the fully relaxed unit cell shows some increase of the lattice constants in GZO compared with $\mathrm{ZnO}$ (see Table III). We considered a very high concentration of $\mathrm{Ga}$ in $\mathrm{ZnO}$ that is at the alloying level (the $\mathrm{Ga}$ atomic concentration is about $6 \%$ in our model). Consequently, the observed increase of the lattice constant should be related to the alloying effect.

Another important observation results from the comparison between measured and calculated $\mathrm{x}$-ray diffraction (XRD) spectra. The predicted XRD spectra show a shift of $\Delta 2 \theta=-0.15 \mathrm{deg}$ corresponding to the (002) reflex. This shift is comparable to the experimentally measured value of $(\Delta 2 \theta=-0.1 \mathrm{deg})$ obtained on nanocrystalline

TABLE III. Calculated and measured (in parentheses) lattice parameters of bulk $\mathrm{ZnO}$ and GZO.

\begin{tabular}{lcc}
\hline \hline & $\mathrm{ZnO}$ & $\mathrm{GZO}$ \\
\hline$a(\AA)$ & $3.213\left(3.25^{a}\right)$ & 3.296 \\
$c(\AA)$ & $5.185\left(5.205^{a}\right)$ & 5.294 \\
$a / c$ & $1.614\left(1.602^{a}\right)$ & 1.607 \\
$2 \theta(\mathrm{deg})$ & $36.65\left(34.38^{b}\right)$ & $36.50\left(34.26^{b}\right)$ \\
$\Delta 2 \theta(\mathrm{deg})$ & $\mathrm{N} / \mathrm{A}$ & $-0.15\left(-0.12^{b}\right)$ \\
\hline \hline
\end{tabular}

${ }^{\mathrm{a}}$ Ref. [32].

${ }^{\mathrm{b}}$ This work. heavy doped (up to 6 at.\%) GZO samples fabricated in this work (see Table III). We can state a good agreement between measured and calculated data. Note that our theoretical model does not account for any contributions of the structural disorder presented in nanocrystalline samples that may cause an observed disagreement between calculated and measured data. Therefore, within our approach the reported effect could be understood as a consequence of the unit-cell changes that may result in structural phase transformation with a further increase of $\mathrm{Ga}$ concentration.

\section{A. Density of states}

The calculated total densities of states (TDOS) of both bulk and Ga-doped $\mathrm{ZnO}$ crystals are shown in Fig. 6. Both curves are normalized with respect to the Fermi energy.

The DOS spectra of the bulk $\mathrm{ZnO}$ show the fully occupied $\mathrm{Zn} 3 d$ semicore levels that are centered at about $7 \mathrm{eV}$ below the valence-band maximum, in good agreement with photoemission measurement showing that the energy of maximum is $-7.4 \mathrm{eV}$ [33]. The upper valence band of $\mathrm{ZnO}$ has a predominant $p$-like character dominated by the O $2 p$ orbitals, and the bottom of the conduction band is $s$ like and is determined by the $\mathrm{Zn} 4 s$ orbitals.

Doping with Ga substantially modifies the density of states, generating additional occupied defect bands at and above the top of the $v$ band (see Fig. 6). At such a very high doping concentration, these states form a new valence-band maximum that will be suitable for increasing the hole concentration. Location of this maximum is Ga-concentration dependent, which can be seen in Fig. 6. However, no remarkable difference in TDOS has been noticed between 3 and $6 \mathrm{wt} \%$ doped GZO, in agreement with the optical spectra measured at different concentrations (see Fig. 3). The energy shift and redistribution of the TDOS with increasing $\mathrm{Ga}$ concentration cause the observed variations in dielectric function spectra (see Fig. 3). Based on the

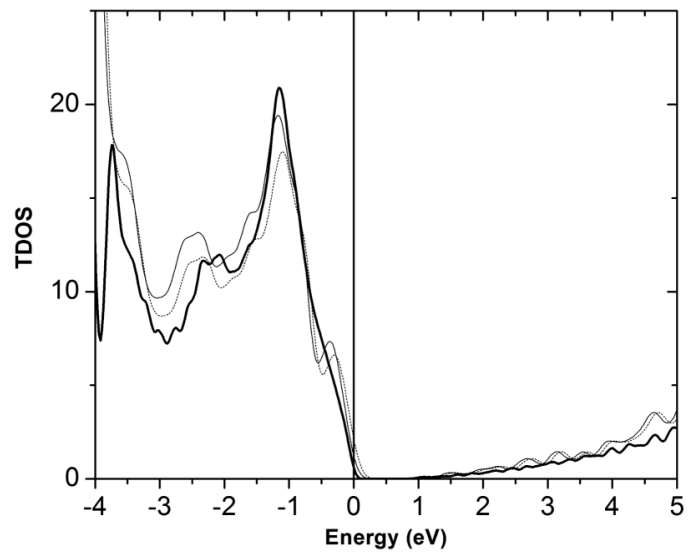

FIG. 6. Total density of states of bulk (bold line) and GZO crystal of nearly $6 \%$ (thin solid line) and $3 \%$ Ga concentration (thin dashed line). 
results of this work, these changes in optical spectra are due to the rehybridization of electronic states followed by the geometry changes with increased $\mathrm{Ga}$ content.

Calculated band-gap values (without corrections) are $E_{g}=$ $0.70 \mathrm{eV}$ and $E_{g}=0.54 \mathrm{eV}$ in undoped and Ga-doped crystals. Analysis of the total and angular-resolved DOS (see Fig. 6) indicates that the lowering of the band gap (by $\Delta E_{g}=-0.16 \mathrm{eV}$ ) is caused by the modifications (rehybridization and renormalization) of electronic orbitals in both valence and conduction bands, as well as structural reconstruction. Comparison between DOS data obtained with unrelaxed and relaxed unit cells indicates a substantial structural reconstruction effect, which we discuss before considering the XRD data (see Table III and discussion above).

\section{B. Optical functions}

It should be noted that the DFT theory version used in this work produces the Kohn-Sham eigenenergy states that do not have a direct interpretation as the one-particle electron energy states [27]. The calculated Kohn-Sham gaps are substantially lower than measured (the "gap problem"). Consequently, the quasiparticle (QP) corrections should be introduced into the theory in order to improve comparison with experiment.

In order to compare calculated optical spectra to the measured data, the QP correction in the electronic structure model that compensates the substantial gap underestimate must be incorporated $[27,31,32]$. In this work, we have obtained the value of the scissor-operator correction $\left(\Delta_{s c i}\right)$ from a comparison with experiment.

The calculated dielectric functions are given in Fig. 7 (for perpendicular $o$ and parallel $e$ polarizations). Our results are compared with experimental data obtained in bulk $\mathrm{ZnO}$ using the generalized ellipsometry by Jellison and

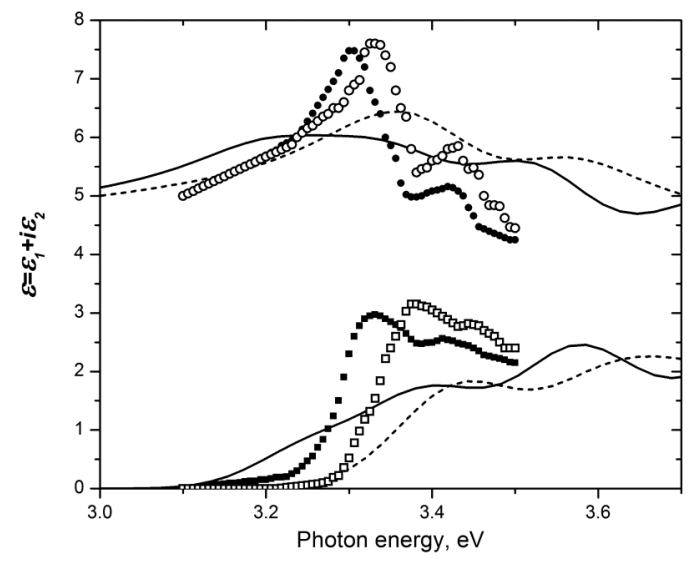

FIG. 7. Real (top of figure) and imaginary (bottom of figure) parts of the dielectric function spectra of $\mathrm{w}-\mathrm{ZnO}$ bulk crystal calculated (lines) and measured (symbols) for ordinary (solid line, filled symbols) and extraordinary light (dashed line, opened symbols). Experimental data were obtained by generalized ellipsometry in Ref. [34].
Boatner [34]. From the comparison, we determined the $\Delta_{\text {sci }}=2.55 \mathrm{eV}$ value that is used to correct the calculated spectra prior to comparing with experimental results.

Our value of $\Delta_{\text {sci }}=2.55 \mathrm{eV}$ is comparable to the $2.70 \mathrm{eV}$ used by Gori et al. [31]. From the comparison between calculated and measured data on bulk $\mathrm{ZnO}$, we can state that our theory (DFT + Sci) correctly reproduces the measured optical anisotropy in wurtzite $\mathrm{ZnO}$ (see the shift between ordinary and extraordinary optical constant spectra in Fig. 7). On the other hand, this version of the theory fails to reproduce a strong peak at the absorption edge. This peak is interpreted in the literature as a consequence of a strong exciton interaction [31,35]. Inclusion of exciton effects in the band-structure calculations requires a numerical solution of the Bethe-Salpeter equation [27,28], which is very computationally expensive and out of the scope of the present work. However, as shown in this work, neglecting the exciton interaction in $\mathrm{ZnO}$ does not preclude us from drawing conclusions about the mechanisms that govern the optical response in doped $\mathrm{ZnO}$.

Further, we consider the mechanisms that change the band gap of $\mathrm{ZnO}$ upon heavy doping. The measured effect of Ga doping on the optical absorption edge of GZO films is shown in Fig. 3. The contributions of plasma excitation to the dielectric function of GZO films with 3 and $6 \mathrm{wt} \%$ doping in the visible and near-infrared spectral regions may be observed. The variations observed are accompanied by substantial blueshifts in the optical absorption edge (see Fig. 3). The following are the main mechanisms that contribute to the optical response of such polycrystalline heavily doped semiconductor films [23]:

i. Burstein-Moss (BM) shift

ii. Atomic structure distortions and reconstructions

iii. Alloying effect, the electron orbital rehybridization, and transformation towards a new solid phase

iv. Exciton effects

In this paper, we focus mostly on the changes in electron energy structure caused by heavy Ga doping. The exciton contributions are neglected because they result in an underestimation of the absolute values of $\epsilon=\epsilon_{1}+i \epsilon_{2}$ and the absence of the characteristic peak at the fundamental absorption edge.

The BM shift describes the blueshift of the optical gap with free-carrier concentration $(n)$ according to $[29,36]$

$$
\Delta E_{g}=\frac{h^{2}}{8 m^{*}}\left(\frac{3}{\pi} n\right)^{\frac{2}{3}}
$$

In order to understand the contribution of the dopinginduced free carriers on the optical spectra in the fundamental absorption region, we calculated $\Delta E_{g}$ according to Eq. (7) and used it as an additional shift, together with $\Delta_{\text {sci }}$ determined for bulk $\mathrm{ZnO}$ (see above). Using data given in Table II for our samples, we obtained $\Delta E_{g}=0.38$ and $0.52 \mathrm{eV}$ for $3 \%$ and $6 \%$ Ga-doped samples, respectively. 
The last value gives the scissors operator equal to $\Delta_{\text {sci }}=$ $3.07 \mathrm{eV}$ for $6 \%$ doped GZO.

The values of $\Delta_{\text {sci }}$ obtained for doped GZO samples are used to compare the calculated $\epsilon(\omega)$ spectra with experiment. In Fig. 8, the calculated dielectric functions are shown in comparison with experimental data for real and imaginary parts of $\epsilon(\omega)$.

We state a good overall agreement between the measured data and the results of the first-principles theory. The plasma contribution has been introduced in the theoretical model by adding the Drude term according to Eq. (5), with parameters determined experimentally (see Table II). Theoretical spectra correctly represent the shapes of the $\epsilon_{1}$ and $\epsilon_{2}$ curves.

Several important issues can be extracted from a comparison between calculated and measured data. There is a substantial lowering of the measured real-part values of the

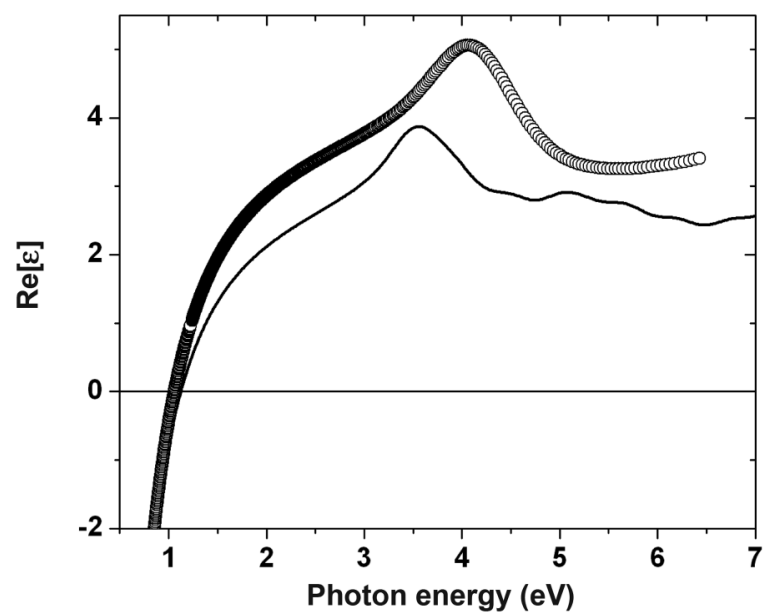

(a)

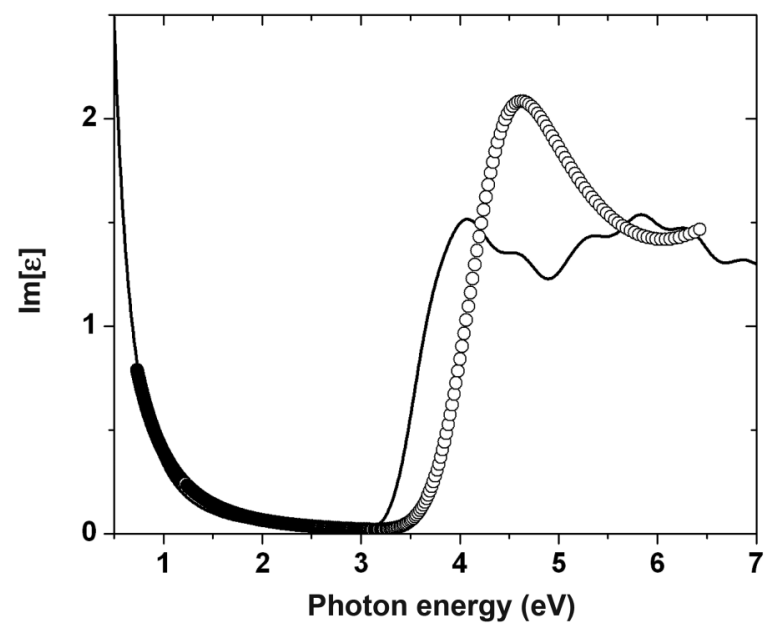

(b)

FIG. 8. Real (a) and imaginary (b) parts of the dielectric function spectra of GZO samples calculated (lines) and measured (symbols) for $6 \mathrm{wt} \%$. dielectric function as a result of Ga doping (see Fig. 8). Our calculated absolute values of $\operatorname{Re}[\epsilon]$ are lower than experimental data. The underestimation of dielectric permittivity values by first-principles theory is caused by using the exchange and correlation method and by neglecting the exciton effects described in the literature [27,28,37]. The reduction of $\epsilon_{1}$ with doping is a result of the electronenergy-structure modification that includes rehybridization of electronic orbitals forming the top of the valence band and the bottom of the conduction band (see above).

Comparison between theory and experiment indicates a strong blueshift in dielectric-function spectra with an increase of $\mathrm{Ga}$ concentration that is substantially higher than the BM shift expected for the given Ga concentrations. The most significant difference between theory and experiment occurs at the highest-studied Ga concentration (see Fig. 8). The bulk values of $\Delta_{\text {sci }}=2.55 \mathrm{eV}$ used to calculate theoretical $\epsilon(\omega)$ spectra are corrected on the BM shift only (0.52 eV for 6\% doping). Using a calculated value of $E_{g}=$ $0.54 \mathrm{eV}$ (see above), this results in a predicted energy gap of $3.61 \mathrm{eV}$, which is substantially lower than the value of $3.89 \mathrm{eV}$ measured here (see Fig. 5). Thus, the measured $\epsilon(\omega)$ spectra are remarkably blueshifted compared to the theory, as shown in Fig. 8. This clearly indicates that the BM effect is not the only mechanism responsible for the blueshift of $\epsilon(\omega)$ spectra with Ga doping.

Changes in the band structure and optical functions with the increase of the component concentration is a wellknown effect in solid alloys [38]. An increase of the Ga concentration should convert GZO into $\mathrm{Ga}_{2} \mathrm{O}_{3}: \mathrm{Zn}$-doped oxide, i.e., through the phase transition. The crystalline $\beta-\mathrm{Ga}_{2} \mathrm{O}_{3}$ oxide has a monoclinic crystal structure and an optical gap in the ultraviolet region, near $4.7 \mathrm{eV}[39,40]$. It is therefore expected that the quasiparticle correction term $\Delta_{\text {sci }}$ is concentration dependent, and it will increase with the Ga concentration. Our theory does not account for any structural transformations corresponding to the phase transition. We use the super-unit-cell approach with the super-cell symmetry corresponding to that of the host $\mathrm{ZnO}$. In such a way, the orbital rehybridization (alloying) effects are partially included in our model, and our results demonstrate that alloying effects are obviously important, even at only a few percent of the Ga concentration. A more advanced model should be assembled, considering possible stable (or metastable) structural phases. This highlights further developments of the GZO optics theory.

In addition, we note that both our experimental and theoretical results of the atomic structure study indicate a substantial increase of the interplanar distance in the doped samples that supports the structural mechanism contributing to the change in the optical functions with doping. The substantial underestimation of the calculated blueshift of the absorption band edge in $6 \mathrm{wt} \%$ GZO films against the measured value is another factor that indicates the 
importance of the structural transformation effect, which should be accounted for by a modified theory.

\section{CONCLUSIONS}

We present the results of experimental and theoretical studies on the optical dielectric functions of gallium-doped $\mathrm{ZnO}$ polycrystalline films that are used as a low-loss plasmonic material. The comparison of dielectric functions of heavily doped GZO films extracted from ellipsometry measurements against those calculated using firstprinciples DFT showed that alloying effects have a significant influence on the optical properties of the films. It was shown that alloying effects are significant, even at relatively low Ga concentrations of $1 \mathrm{wt} \%$. Thus, GZO that is used as a low-loss plasmonic material is not just Gadoped $\mathrm{ZnO}$ but a new alloy material altogether. It was observed that a more sophisticated model is necessary to capture these alloying effects in plasmonic GZO films. This study has provided an insight into understanding the doping mechanism of heavily doped $\mathrm{ZnO}$, which may be applied to other TCOs as well. Understanding the relationship between material, structural, and optical properties, as well as the physical mechanism of doping and its influence on the electronic band structure of these materials, is important for designing low-loss alternative plasmonic materials. This research direction has the potential of eventually solving the inverse-design problem of designing ultimate low-loss plasmonic building blocks that can pave the way for plasmonics and metamaterials being the nextgeneration technologies.

\section{ACKNOWLEDGMENTS}

The authors acknowledge M. Noginov for helpful discussions. The Purdue team for this work is supported by ONR MURI N00014-10-1-0942 and NSF PREM DRM0611430; the team at Norfolk State University is supported by NSF PREM DRM-0611430, AFOSR FA9550-09-10456, and NSF CREST-1036494. O. J. G. and S. M. P., from the Naval Research Laboratory, are supported by ONR Contract No. N0001412WX20514, administered by Dr. Mark Spector.

[1] D. Smith, J. Pendry, and M. Wiltshire, Metamaterials and Negative Refractive Index, Science 305, 788 (2004).

[2] J. Pendry, D. Schurig, and D. Smith, Controlling Electromagnetic Fields, Science 312, 1780 (2006).

[3] H. Atwater, The Promise of Plasmonics, Sci. Am. 296, 56 (2007).

[4] W. Cai and V. Shalaev, Optical Metamaterials: Fundamentals and Applications (Springer Verlag, New York, 2009).

[5] A. Boltasseva and H. Atwater, Low-Loss Plasmonic Metamaterials, Science 331, 290 (2011).
[6] J. Khurgin and G. Sun, In Search of the Elusive Lossless Metal, Appl. Phys. Lett. 96, 181102 (2010).

[7] G. V. Naik, V. M. Shalaev, and A. Boltasseva, Alternative Plasmonic Materials: Beyond Gold and Silver, Adv. Mater. 25, 3264 (2013).

[8] G. Naik and A. Boltasseva, Semiconductors for Plasmonics and Metamaterials, Phys. Status Solidi-R 4, 295 (2010).

[9] C. Rhodes, S. Franzen, J. Maria, M. Losego, D. Leonard, B. Laughlin, G. Duscher, and S. Weibel, Surface Plasmon Resonance in Conducting Metal Oxides, J. Appl. Phys. 100, 054905 (2006).

[10] G. Naik, J. Kim, and A. Boltasseva, Oxides and Nitrides as Alternative Plasmonic Materials in the Optical Range, Opt. Mater. Express 1, 1090 (2011).

[11] M. Noginov, L. Gu, J. Livenere, G. Zhu, A. Pradhan, R. Mundle, M. Bahoura, Y. Barnakov, and V. Podolskiy, Transparent Conductive Oxides: Plasmonic Materials for Telecom Wavelengths, Appl. Phys. Lett. 99, 021101 (2011).

[12] T. R. Gordon, T. Paik, D. R. Klein, G. V. Naik, H. Caglayan, A. Boltasseva, and C. B. Murray, Shape Dependent Plasmonic Response and Directed Self Assembly in a New Semiconductor Building Block, Indium Doped Cadmium Oxide (ICO), Nano Lett. 13, 2857 (2013).

[13] J. Kim, G. Naik, N. Emani, U. Guler, and A. Boltasseva, Plasmonic Resonances in Nanostructured Transparent Conducting Oxide Films, IEEE J. Sel. Top. Quantum Electron. 19, 4601907 (2013).

[14] P. B. Johnson and R. Christy, Optical Constants of the Noble Metals, Phys. Rev. B 6, 4370 (1972).

[15] P. West, S. Ishii, G. Naik, N. Emani, V. Shalaev, and A. Boltasseva, Searching for Better Plasmonic Materials, Laser Photonics Rev. 4, 795 (2010).

[16] M. G. Blaber, M. D. Arnold, and M. J. Ford, Designing Materials for Plasmonic Systems: The Alkali-Noble Intermetallics, J. Phys. Condens. Matter 22, 095501 (2010).

[17] D. A. Bobb, G. Zhu, M. Mayy, A. V. Gavrilenko, P. Mead, V. I. Gavrilenko, and M. A. Noginov, Engineering of LowLoss Metal for Nanoplasmonic and Metamaterials Applications, Appl. Phys. Lett. 95, 151102 (2009).

[18] M. Yoon, S. Lee, H. Park, H. Kim, and M. Jang, Solid Solubility Limits of $\mathrm{Ga}$ and $\mathrm{Al}$ in $\mathrm{ZnO}$, J. Mater. Sci. Lett. 21, 1703 (2002).

[19] H. Kim, C. Gilmore, A. Pique, J. Horwitz, H. Mattoussi, H. Murata, Z. Kafafi, and D. Chrisey, Electrical, Optical, and Structural Properties of Indium-Tin-Oxide Thin Films for Organic Light-Emitting Devices, J. Appl. Phys. 86, 6451 (1999).

[20] K. Postava, H. Sueki, M. Aoyama, T. Yamaguchi, K. Murakami, and Y. Igasaki, Doping Effects on Optical Properties of Epitaxial ZnO Layers Determined by Spectroscopic Ellipsometry, Appl. Surf. Sci. 175, 543 (2001).

[21] F. Shan and Y. Yu, Optical Properties of Pure and Al Doped ZnO Thin Films Fabricated with Plasma Produced by Excimer Laser, Thin Solid Films 435, 174 (2003).

[22] S. M. Park, T. Ikegami, and K. Ebihara, Effects of Substrate Temperature on the Properties of Ga-Doped ZnO by Pulsed Laser Deposition, Thin Solid Films 513, 90 (2006).

[23] V. I. Gavrilenko, Optics of Nanomaterials (Pan Stanford Publishing, Singapore, 2011).

[24] P. D. Paulson, R. W. Birkmire, and W. N. Shafarman, Optical Characterization of $\mathrm{CuIn}_{1-x} \mathrm{Ga}_{x} \mathrm{Se}_{2}$ Alloy Thin 
Films by Spectroscopic Ellipsometry, J. Appl. Phys. 94, 879 (2003).

[25] Y. Kajikawa, Texture Development of Non-epitaxial Polycrystalline ZnO Films, J. Cryst. Growth 289, 387 (2006).

[26] G. Dresselhaus, Optical Absorption Band Edge in Anisotropic Crystals, Phys. Rev. 105, 135 (1957).

[27] R. M. Martin, Electronic Structure. Basic Theory and Practical Methods (Cambridge University Press, Cambridge, England, 2004).

[28] V. I. Gavrilenko and F. Bechstedt, Optical Functions of Semiconductors Beyond Density Functional Theory, Phys. Rev. B 55, 4343 (1997).

[29] J H. Noh, H. S. Jung, J.-K. Lee, J. Y. Kim, C. M. Cho, J.-S. An, and K. S. Hong, Reversible Change in Electrical and Optical Properties in Epitaxially Grown Al-Doped $\mathrm{ZnO}$ Thin Films, Appl. Phys. Lett. 104, 073706 (2008).

[30] M. Usuda, N. Hamada, T. Kotani, and M. van Schilfgaarde, All Electron GW Calculation Based on the LAPW Method: Application to wurtzite ZnO, Phys. Rev. B 66, 125101 (2002).

[31] P. Gori, M. Rakel, C. Cobert, W. Richter, N. Esser, A. Hoffmann, R. D. Sole, A. Cricenti, and O. Pulci, Optical Spectra of $\mathrm{ZnO}$ in the Far Ultraviolet: First- Principles Calculations and Ellipsometric Measurements, Phys. Rev. B 81, 125201 (2010).

[32] X. M. Duan, C. Stampfl, M. M. M. Bilek, and D. R. McKenzie, Codoping of Aluminum and Galium with Nitrogen in ZnO: A Comparative First-Principles Iinvestigation, Phys. Rev. B 79, 235208 (2009).
[33] A. R. H. Preston, B. J. Ruck, L. F. J. Piper, A. DeMasi, K. E. Smith, A. Schleife, F. Fuchs, F. Bechstedt, J. Chai, and S. M. Durbin, Band Structure of $\mathrm{ZnO}$ from Resonant $X$-Ray Emission Spectroscopy, Phys. Rev. B 78, 155114 (2008).

[34] J. G. E. Jellison and L. A. Boatner, Optical Functions of Uniaxial ZnO Determined by Generalized Ellipsometry, Phys. Rev. B 58, 3586 (1998).

[35] J. F. Muth, R. M. Kolbas, A. K. Sharma, S. Oktyabrsky, and J. Narayan, Excitonic Structure and Absorption Coeffecient Measurements of $\mathrm{ZnO}$ Single Crystal Epitaxial Films Deposited by Pulsed Laser Deposition, J. Appl. Phys. 85, 7884 (1999).

[36] E. Burstein, Anomalous Optical Absorption Limit in InSb, Phys. Rev. 93, 632 (1954).

[37] V. I. Gavrilenko, in Tutorials in Complex Photonic Media, edited by M. A. Noginov, M. W. McCall, G. Dewar, and N. I. Zheludev (SPIE Press, Bellingham, 2009), Chap. 15, pp. 479-524.

[38] I. Vurgaftman, J. R. Meyer, and L. R. Ram-Mohan, Band Parameters for III-V Compound Semiconductors and Their Alloys, J. Appl. Phys. 89, 5815 (2001).

[39] M. Yamaga, E. G. Víllora, K. Shimamura, N. Ichinose, and M. Honda, Donor Structure and Electric Transport Mechanism in $\beta-\mathrm{Ga}_{2} \mathrm{O}_{3}$, Phys. Rev. B 68, 155207 (2003).

[40] Y. Zhang, J. Yan, G. Zhao, and W. Xie, First-Principles Study on Electronic Structure and Optical Properties of Sn-doped $\beta-\mathrm{Ga}_{2} \mathrm{O}_{3}$, Physica (Amsterdam) 405B, 3899 (2010). 